Research Article

\title{
Random Attractors of Stochastic Three-Component Reversible Gray-Scott System on Unbounded Domains
}

\author{
Anhui Gu \\ College of Science, Guilin University of Technology, Guangxi, Guilin 541004, China \\ Correspondence should be addressed to Anhui Gu, mathgu@yahoo.cn \\ Received 26 May 2012; Revised 20 July 2012; Accepted 23 July 2012 \\ Academic Editor: Norio Yoshida \\ Copyright (c) 2012 Anhui Gu. This is an open access article distributed under the Creative \\ Commons Attribution License, which permits unrestricted use, distribution, and reproduction in \\ any medium, provided the original work is properly cited. \\ The existence of a pullback random attractor is established for a stochastic three-component \\ reversible Gray-Scott system on unbounded domains. The Gray-Scott system is recast as a random \\ dynamical system and asymptotic compactness which is illustrated by using uniform, a priori \\ estimates for far-field values of solutions and a cutoff technique.
}

\section{Introduction}

Consider the asymptotic behavior of solutions of the following stochastic three-component reversible Gray-Scott system with multiplicative noise defined in the entire space $\mathbb{R}^{n} \times \mathbb{R}^{n} \times \mathbb{R}^{n}$ :

$$
\begin{aligned}
& \frac{\partial \tilde{u}}{\partial t}=d_{1} \Delta \tilde{u}-(F+k) \tilde{u}+\tilde{u}^{2} \tilde{v}-G \tilde{u}^{3}+N \tilde{w}+f_{1}(x)+\sigma \tilde{u} \circ \frac{d B_{t}}{d t}, \\
& \frac{\partial \tilde{v}}{\partial t}=d_{2} \Delta \tilde{v}-F \widetilde{v}-\tilde{u}^{2} \widetilde{v}+G \tilde{u}^{3}+f_{2}(x)+\sigma \widetilde{v} \circ \frac{d B_{t}}{d t}, \\
& \frac{\partial \tilde{w}}{\partial t}=d_{3} \Delta \tilde{w}-(F+N) \tilde{w}+k \tilde{u}+f_{3}(x)+\sigma \widetilde{w} \circ \frac{d B_{t}}{d t},
\end{aligned}
$$

with initial data

$$
\tilde{u}(0, x)=\tilde{u}_{0}(x), \quad \tilde{v}(0, x)=\widetilde{v}_{0}(x), \quad \tilde{w}(0, x)=\widetilde{w}_{0}(x), \quad x \in \mathbb{R}^{n},
$$


where all the parameters are given positive constants; $f_{i}(i=1,2,3)$ are nonlinear functions satisfying certain conditions; $B_{t}$ is a two-sided real-valued Wiener process on a probability space $(\Omega, \mathcal{F}, \mathbb{P}), \Omega=\{\omega \in C(\mathbb{R}, \mathbb{R}): \omega(0)=0\}$, the Borel $\sigma$-algebra $\mathcal{F}$ on $\Omega$ is generated by the compact open topology (see [1]), and $\mathbb{P}$ is the corresponding Wiener measure on $\mathcal{F}$; $\circ$ denotes the Stratonovich sense of the stochastic term.

Historically, when $\tilde{w}=0, G=0, f_{1}=f_{3}=0, f_{2}=F$ and there are no random terms $(\sigma=0)$, system (1.1) reduces to the two-component Gray-Scott system which signified one of the Brussels schools led by the renowned physical chemist and Nobel Prize laureate (1977), Ilya Prigogine, which originated from describing an isothermal, cubic autocatalytic, continuously fed, and diffusive reactions of two chemicals (see [2-6]). The three-component reversible Gray-Scott model was firstly introduced by Mahara et al., which is based on the scheme of two reversible chemical or biochemical reactions [7]. Then in [8], You took some nondimensional transformations, the three-component reversible system was reduced to the system (1.1) without random forces. In [8], You considered the existence of global attractor for the system (1.1) with Neumann boundary condition on a bounded domain of space dimension $n \leq 3$ by the method of the rescaling and grouping estimation.

Stochastic differential equations of this type arise from many chemical or biochemical systems when random spatiotemporal forcing is taken into consideration. These random perturbations are intrinsic effects in a variety of settings and spatial scales. They may be most obviously influential at the microscopic and smaller scales, but indirectly they play an important role in macroscopic phenomena. Recently, Gu [9] gave the existence of a compact random attractor for stochastic three-component reversible Gray-Scott system with multiplicative white noise in a bounded domain of $\mathbb{R}^{n}(n \leq 3)$ when $f_{1}=f_{3}=0, f_{2}=F$ in system (1.1). As pointed in [10], the discussion of the same or similar coupled reactiondiffusion systems on a higher dimensional domain with the space dimension $n>3$ and on an unbounded domain is still open to the best of our knowledge. Here, we intend to investigate the dynamical behavior of the system (1.1) on unbounded domains and give a partly answer to the problems proposed in [10]. It is worth mentioning that Sobolev embedding is not compact on domains of infinite volume. This introduces a major obstacle for proving the existence of random attractors for partial differential equations on unbounded domains. For some deterministic equations, the difficulty caused by the unboundedness of domains can be overcome by the energy equation approach which developed by Ball in [11, 12] and used by many authors (see, e.g., [13-15]). In this paper, we will use the uniform estimates on the far-field values of solutions to circumvent the difficulty caused by the unboundedness of the domain. This idea was developed in [16] to prove the asymptotic compactness of solutions for autonomous parabolic equations on $\mathbb{R}^{n}$, and later extended to nonautonomous equations (see, e.g., [17-19]) and stochastic equations (see, e.g., [20-22]). Here, we will use the method of tail-estimates to investigate the asymptotic behavior of system (1.1) with initial data (1.2).

The paper is organized as follows. In the next section, we recall the fundamental concepts and results for pullback random attractors for random dynamical systems. In Section 3, we define a cocycle for the stochastic three-component reversible Gray-Scott system on $\mathbb{R}^{n} \times \mathbb{R}^{n} \times \mathbb{R}^{n}$. Section 4 is devoted to deriving the uniform estimates of solutions for large space and time variables. In the last section, we give the asymptotic compactness of the solution by using uniform estimates on the tails of solutions and then prove the existence of a pullback random attractor.

The following notations will be used throughout the paper. We denote by $\|\cdot\|$ and $(, \cdot$, the norm and inner product in $L^{2}\left(\mathbb{R}^{n}\right)$ or $\mathbb{H}=\left[L^{2}\left(\mathbb{R}^{n}\right)\right]^{3}$. Let $\mathbb{U}=\left[L^{6}\left(\mathbb{R}^{n}\right)\right]^{3} ; \mathbb{E}=\left[H^{1}\left(\mathbb{R}^{n}\right)\right]^{3} ;$ $\|\cdot\|_{L^{6}}$ and $\|\cdot\|_{\mathbb{U}}$ denote the norm in $L^{6}\left(\mathbb{R}^{n}\right)$ and $\mathbb{U}$. 


\section{Preliminaries}

In this section, we recall some basic concepts related to random attractors for random dynamical systems. We refer the reader to $[1,23,24]$ for more details.

Let $\left(X,\|\cdot\|_{X}\right)$ be a separable Hilbert space with Borel $\sigma$-algebra $B(X)$, and let $(\Omega, \mathcal{F}, \mathbb{P})$ be a probability space.

Definition 2.1. $\left(\Omega, \mathcal{F}, \mathbb{P},\left(\theta_{t}\right)_{t \in \mathbb{R}}\right)$ is called a metric dynamical system if $\theta: \mathbb{R} \times \Omega \mapsto \Omega$ is $(\mathbb{B}(\mathbb{R}) \times$ $\mathcal{F}, \mathcal{F}$ )-measurable and $\theta_{0}$ is the identity on $\Omega, \theta_{s+t}=\theta_{t} \theta_{s}$ for all $s, t \in \mathbb{R}$ and $\theta_{t} \mathbb{P}=\mathbb{P}$ for all $t \in \mathbb{R}$.

Definition 2.2. A stochastic process $\{\varphi(t, \omega)\}_{t \geq 0, \omega \in \Omega}$ is a continuous random dynamical system (RDS) over $\left(\Omega, \mathcal{F}, \mathbb{P},\left(\theta_{t}\right)_{t \in \mathbb{R}}\right)$ if $\varphi$ is $(\mathbb{B}[0, \infty) \times \mathscr{F} \times \mathbb{B}(X), \mathbb{B}(X))$-measurable, and for all $\omega \in \Omega$,

(i) the mapping $\varphi(t, \omega): X \mapsto X, x \mapsto \varphi(t, \omega) x$ is continuous for every $t \geq 0$;

(ii) $\varphi(0, \omega)$ is the identity on $X$;

(iii) (cocycle property) $\varphi(s+t, \omega)=\varphi\left(t, \theta_{s} \omega\right) \varphi(s, \omega)$ for all $s, t \geq 0$.

Definition 2.3. (i) A set-valued mapping $\omega \mapsto B(\omega) \subset X$ (we may write it as $B(\omega)$ for short) is said to be a random set if the mapping $\omega \mapsto \operatorname{dist}_{X}(x, B(\omega))$ is measurable for any $x \in X$.

(ii) A random set $B(\omega)$ is said to be bounded if there exist $x_{0} \in X$ and a random variable $r(\omega)>0$ such that $B(\omega) \subset\left\{x \in X:\left\|x-x_{0}\right\|_{X} \leq r(\omega), x_{0} \in X\right\}$ for all $\omega \in \Omega$.

(iii) A random set $B(\omega)$ is called a compact random set if $B(\omega)$ is compact for all $\omega \in \Omega$.

(iv) A random bounded set $B(\omega) \subset X$ is called tempered with respect to $\left(\theta_{t}\right)_{t \in \mathbb{R}}$ if for a.e. $\omega \in \Omega \lim _{t \rightarrow+\infty} e^{-\gamma t} \sup _{x \in B\left(\theta_{-t} \omega\right)}\|x\|_{X}=0$ for all $\gamma>0$. A random variable $\omega \mapsto r(\omega) \in \mathbb{R}$ is said to be tempered with respect to $\left(\theta_{t}\right)_{t \in \mathbb{R}}$ if for a.e. $\omega \in \Omega, \lim _{t \rightarrow+\infty} e^{-\gamma t} \sup _{t \in \mathbb{R}}\left|r\left(\theta_{-t} \omega\right)\right|=$ 0 for all $\gamma>0$.

We consider a continuous RDS $\{\varphi(t, \omega)\}_{t \geq 0, \omega \in \Omega}$ over $\left(\Omega, \mathcal{F}, \mathbb{P},\left(\theta_{t}\right)_{t \in \mathbb{R}}\right)$ and $\boldsymbol{\Phi}$ the set of all tempered random sets of $X$.

Definition 2.4. A random set $\mathcal{K}(\omega)$ is called an absorbing set in $\Phi$ if for all $B \in \Phi$ and a.e. $\omega \in \Omega$ there exist $t_{B}(\omega)>0$ such that

$$
\varphi\left(t, \theta_{-t} \omega\right) B\left(\theta_{-t} \omega\right) \subset \mathcal{K}(\omega) \quad \forall t \geq t_{B}(\omega)
$$

Definition 2.5. A random set $\mathbb{A}$ is called a global $\boldsymbol{\Phi}$-random attractor (or $\boldsymbol{\Phi}$-pullback attractor) for $\{\varphi(t, \omega)\}_{t \geq 0, \omega \in \Omega}$ if the following hold:

(i) $\mathcal{A}$ is a random compact set, that is $\omega \mapsto d(x, \mathcal{A}(\omega))$ is measurable for every $x \in X$ and $\mathcal{A}(\omega)$ is compact for a.e. $\omega \in \Omega$;

(ii) $\mathcal{A}$ is strictly invariant, that is, for $\omega \in \Omega$ and all $t \geq 0$ one has $\varphi(t, \omega) \mathcal{A}(\omega)=\mathcal{A}\left(\theta_{t} \omega\right)$;

(iii) $\mathcal{A}$ attracts all sets in $\Phi$, that is, for all $B \in \Phi$ and a.e. $\omega \in \Omega$ we have

$$
\lim _{t \rightarrow+\infty} d\left(\varphi\left(t, \theta_{-t} \omega\right) B\left(\theta_{-t} \omega\right), \mathcal{A}(\omega)\right)=0,
$$

where $d(Y, Z)=\sup _{y \in Y} \inf _{z \in Z}\|y-z\|_{X}$ is the Hausdorff semimetric $(Y \subseteq X, Z \subseteq X)$. 
Proposition 2.6 (see [24]). Let $K(\omega) \in \Phi$ be a random absorbing set for the continuous RDS $(\varphi(t))_{t \geq 0}$, which is closed and satisfies for a.e. $\omega \in \Omega$ the following asymptotic compactness condition: each sequence $x_{n} \in \varphi\left(t_{n}, \theta_{-t_{n}} \omega, K\left(\theta_{-t_{n}} \omega\right)\right)$ with $t_{n} \rightarrow \infty$ has a convergent subsequence in $X$. Then, the cocycle $\varphi$ has a unique global random attractor.

$$
\mathcal{A}(\omega)=\bigcap_{\tau \geq t_{K}(\omega) t \geq \tau} \overline{\bigcup_{\varphi} \varphi\left(t, \theta_{t}(\omega), K\left(\theta_{t}(\omega)\right)\right)}
$$

\section{RDS Generated by Stochastic Three-Component Reversible Gray-Scott System}

In this section, we will give the basic setting of system (1.1) and show that it generates a random dynamical system. Let $(\Omega, \mathcal{F}, \mathbb{P})$ be a probability space as in Section 1 . Define $\left(\theta_{t}\right)_{t \in \mathbb{R}}$ on $\Omega$ via $\theta_{t} \omega(\cdot)=\omega(\cdot+t)-\omega(t), t \in \mathbb{R}$, then $\left(\Omega, \mathcal{F}, \mathbb{P},\left(\theta_{t}\right)_{t \in \mathbb{R}}\right)$ is an ergodic metric dynamical system (see $[1,23])$.

Denote $\widetilde{g}=(\widetilde{u}, \widetilde{v}, \widetilde{w})^{T}$, system (1.1) with initial data (1.2) can be rewritten as

$$
\begin{aligned}
& \frac{\partial \widetilde{g}}{\partial t}=A \widetilde{g}+\tilde{\Lambda}(\tilde{g})+f(x)+\sigma \widetilde{g} \circ \frac{d B_{t}}{d t}, \quad t>0, \\
& \tilde{g}(0, x)=\widetilde{g}_{0}(x), \quad x \in \mathbb{R}^{n},
\end{aligned}
$$

where

$$
A=\left(\begin{array}{ccc}
d_{1} & 0 & 0 \\
0 & d_{2} & 0 \\
0 & 0 & d_{3}
\end{array}\right) \Delta, \quad \tilde{\Lambda}(\tilde{g})=\left(\begin{array}{c}
-(F+k) \tilde{u}+\tilde{u}^{2} \widetilde{v}-G \tilde{u}^{3}+N \widetilde{w} \\
-F \widetilde{v}-\widetilde{u}^{2} \widetilde{v}+G \widetilde{u}^{3} \\
k \tilde{u}-(F+N) \tilde{w}
\end{array}\right)
$$

and $f(x)=\left(f_{1}(x), f_{2}(x), f_{3}(x)\right)^{\mathrm{T}}$, here $\mathbf{T}$ denotes the transposition.

For our purpose, it is convenient to transform the problem (3.1) into a deterministic system with a random parameter and then show that it generates a random dynamical system.

Before performing this transformation, we need to recall some properties of the Ornstein-Uhlenbeck processes. Let

$$
z\left(\theta_{t} \omega\right)=-\int_{-\infty}^{0} e^{\tau} \theta_{t} \omega(\tau) d \tau, \quad t \in \mathbb{R}, \omega \in \Omega
$$

We know that $z\left(\theta_{t} \omega\right)$ is an Ornstein-Uhlenbeck process on $\left(\Omega, \mathcal{F}, \mathbb{P},\left(\theta_{t}\right)_{t \in \mathbb{R}}\right)$ and solves the following one-dimensional stochastic differential equation (see [25] for details):

$$
d z=-z d t+d B_{t}, \quad z(-\infty)=0, \quad \forall t \geq 0, \omega \in \Omega,
$$


where $B_{t}(\omega)=B(t, \omega)=\omega(t)$ for $\omega \in \Omega, t \in \mathbb{R}$. In fact, from $[1,26]$, we know that the random variable $z(\omega)$ is tempered, and there is a $\theta_{t}$-invariant set $\widetilde{\Omega} \subset \Omega$ of full $\mathbb{P}$ measure such that for $\omega \in \tilde{\Omega}, t \mapsto z\left(\theta_{t} \omega\right)$ is continuous in $t$; furthermore,

$$
\lim _{t \rightarrow \pm \infty} \frac{\left|z\left(\theta_{t} \omega\right)\right|}{t}=\lim _{t \rightarrow \pm \infty} \frac{1}{t} \int_{0}^{t} z\left(\theta_{s} \omega\right) d s=0
$$

Let

$$
\begin{aligned}
& u(t)=\alpha(t) \tilde{u}(t) \\
& v(t)=\alpha(t) \tilde{v}(t) \quad \text { with } \alpha(t)=e^{-\sigma z\left(\theta_{t} \omega\right)} \\
& w(t)=\alpha(t) \tilde{w}(t),
\end{aligned}
$$

then system (3.1) can be written as

$$
\begin{gathered}
\frac{\partial u}{\partial t}=d_{1} \Delta u-\left(F+k-\sigma z\left(\theta_{t} \omega\right)\right) u+\alpha^{-2}(t) u^{2} v-G \alpha^{-2}(t) u^{3}+N w+\alpha(t) f_{1} \\
\frac{\partial v}{\partial t}=d_{2} \Delta v-\left(F-\sigma z\left(\theta_{t} \omega\right)\right) v-\alpha^{-2}(t) u^{2} v+G \alpha^{-2}(t) u^{3}+\alpha(t) f_{2} \\
\frac{\partial w}{\partial t}=d_{3} \Delta w-\left(F+N-\sigma z\left(\theta_{t} \omega\right)\right) w+k u+\alpha(t) f_{3}
\end{gathered}
$$

that is, $g(t, \cdot)=(u(t, \cdot), v(t, \cdot), w(t, \cdot)), t>0$ satisfies

$$
\begin{aligned}
& \frac{d g}{d t}=A g+\Lambda(g, \omega)+\alpha(t) f(x), \quad t>0, \\
& g(0, x)=g_{0}(x)=e^{-\sigma z(\omega)} \widetilde{g}_{0}(x), \quad x \in \mathbb{R}^{n},
\end{aligned}
$$

where

$$
\Lambda(g, \omega)=\left(\begin{array}{c}
-\left(F+k-\sigma z\left(\theta_{t} \omega\right)\right) u+\alpha^{-2}(t) u^{2} v-G \alpha^{-2}(t) u^{3}+N w \\
-\left(F-\sigma z\left(\theta_{t} \omega\right)\right) v-\alpha^{-2}(t) u^{2} v+G \alpha^{-2}(t) u^{3} \\
-\left(F+N-\sigma z\left(\theta_{t} \omega\right)\right) w+k u
\end{array}\right)
$$

We will consider (3.8) for $\omega \in \widetilde{\Omega}$ and still use $\Omega$ instead of $\widetilde{\Omega}$ from now on.

As in the case of a bounded domain with Dirichlet boundary conditions which are studied in [27], for $\Lambda: \mathbb{E} \cap \mathbb{U} \mapsto \mathbb{H}$ is locally Lipschitz continuous and $f \in \mathbb{H} \cap \mathbb{U}$, by a Galerkin method, one can show that for $\mathbb{P}$-a.e. $\omega \in \Omega$ and for all $g_{0} \in \mathbb{H}$, (3.8) has a unique solution $g\left(\cdot, \omega, g_{0}\right) \in C([0, \infty), \mathbb{H}) \cap L^{2}((0, T) ; \mathbb{E})$ with $g\left(0, \omega, g_{0}\right)=g_{0}$ for every $T>0$. Similarly to [28], because of the continuous nonlinearity $\Lambda$, one may take the domain to be a sequence of balls with radius approaching $\infty$ to deduce the existence of a weak solution to (3.8) on $\mathbb{R}^{n} \times \mathbb{R}^{n} \times \mathbb{R}^{n}$. Furthermore, one may get that $g\left(t, \omega, g_{0}\right)$ is unique and continuous with respect 
to $g_{0}$ in $\mathbb{H}$ for all $t \geq 0$. Then, (3.8) generates a continuous random dynamical system $(\varphi(t))_{t \geq 0}$ over $\left(\Omega, \mathcal{F}, \mathbb{P},\left(\theta_{t}\right)_{t \in \mathbb{R}}\right)$ according to the conditions (i)-(iii) in Definition 2.2, where

$$
\varphi\left(t, \omega, \tilde{g}_{0}\right)=g\left(t, \omega, g_{0}\right), \quad \forall\left(t, \omega, \tilde{g}_{0}\right) \in \mathbb{R}^{+} \times \Omega \times \mathbb{H} .
$$

We now define a mapping $\phi: \mathbb{R}^{+} \times \Omega \times \mathbb{H} \rightarrow \mathbb{H}$ by

$$
\phi\left(t, \omega, \widetilde{g}_{0}\right)=\tilde{g}\left(t, \omega, \widetilde{g}_{0}\right)=g\left(t, \omega, e^{-\sigma z(\omega)} \widetilde{g}_{0}\right) e^{\sigma z\left(\theta_{t} \omega\right)},
$$

for all $\left(t, \omega, \widetilde{g}_{0}\right) \in \mathbb{R}^{+} \times \Omega \times \mathbb{H}$.

Then $\phi$ is a continuous random dynamical system associated with problem (3.1) on $\mathbb{R}^{n} \times \mathbb{R}^{n} \times \mathbb{R}^{n}$

We remark that the two random dynamical systems are conjugated to each other; thus, the inverse transformation of $\varphi$ is a solution of the original system. For more details on the conjugate theory of stochastic and random differential equations, we can refer to [29]. Thus, in the following sections, we only need to consider the existence of a random attractor of $\varphi$.

\section{Uniform Estimates of Solutions}

In this section, we establish the uniform estimates on the solution of the stochastic Gray-Scott system on $\mathbb{R}^{n} \times \mathbb{R}^{n} \times \mathbb{R}^{n}$ when $t \rightarrow \infty$ in order to derive the existence of a bounded random absorbing set and the asymptotic compactness of the random dynamical system associated with the problem. Particularly when time is sufficiently large, we will show that the tails of the solutions for large space variables are uniformly small.

We always assume that $\Phi$ is the collection of all tempered subsets of $\mathbb{H}$ with respect to $\left(\Omega, \mathcal{F}, \mathbb{P},\left(\theta_{t}\right)_{t \in \mathbb{R}}\right)$. The next lemma implied that $\varphi$ has a random absorbing set in $\boldsymbol{\Phi}$.

Lemma 4.1. Assume that $f \in \mathbb{H}$. Then, there exists a random ball $\{\mathcal{A}(\omega)\} \in \Phi$ centered at 0 with radius $\bar{R}(\omega)>0$ such that $\{\mathcal{A}(\omega)\}$ is a random absorbing set for $\varphi$ in $\Phi$, that is, for any $\{B(\omega)\} \in \Phi$ and $\mathbb{P}$-a.e. $\omega \in \Omega$, there is $T_{B}(\omega)>0$ such that

$$
\varphi\left(t, \theta_{-t} \omega, B\left(\theta_{-t} \omega\right)\right) \subseteq \mathcal{A}(\omega) \quad \forall t>T_{B}(\omega)
$$

Proof. Define

$$
W(t, x)=\frac{N}{k} w(t, x), \quad \mu=\frac{k}{N},
$$

then the system (3.8) becomes

$$
\begin{gathered}
\frac{\partial u}{\partial t}=d_{1} \Delta u-\left(F+k-\sigma z\left(\theta_{t} \omega\right)\right) u+\alpha^{-2}(t) u^{2} v-G \alpha^{-2}(t) u^{3}+k W+\alpha(t) f_{1}, \\
\frac{\partial v}{\partial t}=d_{2} \Delta v-\left(F-\sigma z\left(\theta_{t} \omega\right)\right) v-\alpha^{-2}(t) u^{2} v+G \alpha^{-2}(t) u^{3}+\alpha(t) f_{2}, \\
\mu \frac{\partial W}{\partial t}=\mu d_{3} \Delta W-\left(\mu F+k-\mu \sigma z\left(\theta_{t} \omega\right)\right) W+k u+\alpha(t) f_{3} .
\end{gathered}
$$


Take the inner products $((4.3), G u(t)),((4.4), v(t))$, and $((4.5), G W(t))$. Then summing up the resulting equalities, we get

$$
\begin{aligned}
\frac{d}{d t}\left(G\|u\|^{2}+\|v\|^{2}+\mu G\|W\|^{2}\right)+2 d_{1} G\|\nabla u\|^{2}+2 d_{2}\|\nabla v\|^{2} \\
\quad+2 \mu G d_{3}\|\nabla W\|^{2}+2\left(G(F+k)\|u\|^{2}+F\|v\|^{2}+G(\mu F+k)\|W\|^{2}\right) \\
=4 k G \int_{\mathbb{R}^{n}} u W d x+2 \int_{\mathbb{R}^{n}} \alpha(t) u f_{1} d x+2 \int_{\mathbb{R}^{n}} \alpha(t) v f_{2} d x+2 \int_{\mathbb{R}^{n}} \alpha(t) W f_{3} d x \\
\quad+2 \sigma z\left(\theta_{t} \omega\right)\left(G\|u\|^{2}+\|v\|^{2}+\mu G\|W\|^{2}\right)-2 \alpha^{-2}(t) \int_{\mathbb{R}^{n}}\left(G u^{2}-u v\right)^{2} d x
\end{aligned}
$$

that is,

$$
\begin{aligned}
\frac{d}{d t}\left(G\|u\|^{2}+\|v\|^{2}+\mu G\|W\|^{2}\right)+2 d_{1} G\|\nabla u\|^{2}+2 d_{2}\|\nabla v\|^{2}+2 \mu G d_{3}\|\nabla W\|^{2} \\
\leq\left(2 \sigma z\left(\theta_{t} \omega\right)-F\right)\left(G\|u\|^{2}+\|v\|^{2}+\mu G\|W\|^{2}\right) \\
\quad+\frac{\alpha^{2}(t)}{G F}\left(\left\|f_{1}\right\|^{2}+G\left\|f_{2}\right\|^{2}+\frac{1}{\mu}\left\|f_{3}\right\|^{2}\right) .
\end{aligned}
$$

Let $c_{1}=\max \{1, G, 1 / \mu\} / G F \min \{1, G, 1 / \mu\}$ and apply Gronwall lemma to (4.7) for $t \geq 0$, we have

$$
\begin{aligned}
\left\|g\left(t, \omega, g_{0}(\omega)\right)\right\|^{2} \leq & e^{2 \sigma \int_{0}^{t} z\left(\theta_{s} \omega\right) d s-F t}\left\|g_{0}(\omega)\right\|^{2} \\
& +c_{1}\|f\|^{2} e^{2 \sigma \int_{0}^{t} z\left(\theta_{s} \omega\right) d s-F t} \int_{0}^{t} e^{-2 \sigma z\left(\theta_{s} \omega\right)-2 \sigma \int_{0}^{s} z\left(\theta_{\tau} \omega\right) d \tau+F s} d s .
\end{aligned}
$$

By substituting $\omega$ with $\theta_{-t} \omega$ in (4.8), it yields that

$$
\begin{aligned}
\| g(t, & \left.\theta_{-t} \omega, g_{0}\left(\theta_{-t} \omega\right)\right) \|^{2} \\
\leq & e^{2 \sigma \int_{0}^{t} z\left(\theta_{s-t} \omega\right) d s-F t}\left\|g_{0}\left(\theta_{-t} \omega\right)\right\|^{2} \\
& +c_{1}\|f\|^{2} e^{2 \sigma \int_{0}^{t} z\left(\theta_{s-t} \omega\right) d s-F t} \int_{0}^{t} e^{-2 \sigma z\left(\theta_{s-t} \omega\right)-2 \sigma \int_{0}^{s} z\left(\theta_{\tau-t} \omega\right) d \tau+F s} d s \\
\leq & e^{2 \sigma \int_{-t}^{0} z\left(\theta_{s} \omega\right) d s-F t}\left\|g_{0}\left(\theta_{-t} \omega\right)\right\|^{2} \\
& +c_{1}\|f\|^{2} \int_{-t}^{0} e^{-2 \sigma z\left(\theta_{s} \omega\right)-2 \sigma \int_{s}^{0} z\left(\theta_{\tau} \omega\right) d \tau+F s} d s
\end{aligned}
$$




$$
\begin{aligned}
\leq & e^{2 \sigma \int_{-t}^{0} z\left(\theta_{s} \omega\right) d s-F t}\left\|g_{0}\left(\theta_{-t} \omega\right)\right\|^{2} \\
& +c_{1}\|f\|^{2} \int_{-\infty}^{0} e^{-2 \sigma z\left(\theta_{s} \omega\right)-2 \sigma \int_{s}^{0} z\left(\theta_{\tau} \omega\right) d \tau+F s} d s .
\end{aligned}
$$

By (3.5), we obtain

$$
\int_{-\infty}^{0} e^{-2 \sigma z\left(\theta_{s} \omega\right)-2 \sigma \int_{s}^{0} z\left(\theta_{\tau} \omega\right) d \tau+F s} d s<+\infty
$$

Notice that $B(\omega) \in \Phi$ is tempered, then for any $g_{0}\left(\theta_{-t} \omega\right) \in B\left(\theta_{-t} \omega\right)$,

$$
\lim _{t \rightarrow+\infty} e^{2 \sigma \int_{-t}^{0} z\left(\theta_{s} \omega\right) d s-F t}\left\|g_{0}\left(\theta_{-t} \omega\right)\right\|^{2}=0
$$

Denote

$$
\bar{R}^{2}(\omega)=1+c_{1}\|f\|^{2} \int_{-\infty}^{0} e^{-2 \sigma z\left(\theta_{s} \omega\right)-2 \sigma \int_{s}^{0} z\left(\theta_{\tau} \omega\right) d \tau+F s} d s
$$

then $\{\mathcal{A}(\omega)\} \in \Phi$ is a random absorbing set for $\varphi$ in $\Phi$, which completes the proof.

Lemma 4.2. Assume that $f \in \mathbb{H}$. Then there exists a tempered random variable $\tilde{R}(\omega)>0$ such that for any $B(\omega) \in \Phi$ and $g_{0}(\omega) \in B(\omega)$, there exists a $T_{B}(\omega)>0$ such that the solution $\varphi$ of (3.8) satisfies for $\mathbb{P}$-a.e. $\omega \in \Omega$, for all $t \geq T_{B}(\omega)$,

$$
\int_{t}^{t+1}\left\|\nabla \varphi\left(s, \theta_{-t-1} \omega, g_{0}\left(\theta_{-t-1} \omega\right)\right)\right\|^{2} d s \leq \tilde{R}(\omega)
$$

Proof. By replacing $t$ and $\omega$ with $\tilde{T}$ and $\theta_{-t} \omega$ in (4.8), for $\tilde{T}>0$, we get

$$
\begin{aligned}
& \left\|g\left(\widetilde{T}, \theta_{-t} \omega, g_{0}\left(\theta_{-t} \omega\right)\right)\right\|^{2} \\
& \leq e^{2 \sigma \int_{0}^{\tilde{T}} z\left(\theta_{s-t} \omega\right) d s-F \tilde{T}}\left\|g_{0}\left(\theta_{-t} \omega\right)\right\|^{2} \\
& \quad+c_{1}\|f\|^{2} e^{2 \sigma \int_{0}^{\tilde{T}} z\left(\theta_{s-t} \omega\right) d s-F t} \int_{0}^{\tilde{T}} e^{-2 \sigma z\left(\theta_{s-t} \omega\right)-2 \sigma \int_{0}^{s} z\left(\theta_{\tau-t} \omega\right) d \tau+F s} d s .
\end{aligned}
$$


Multiplying both sides of (4.14) with $e^{2 \sigma \int_{\tilde{T}}^{t} z\left(\theta_{s-t} \omega\right) d s-F(t-\tilde{T})}$, we have for $t \geq \tilde{T}$

$$
\begin{aligned}
& e^{2 \sigma \int_{\tilde{T}}^{t} z\left(\theta_{s-t} \omega\right) d s-F(t-\widetilde{T})}\left\|g\left(\widetilde{T}, \theta_{-t} \omega, g_{0}\left(\theta_{-t} \omega\right)\right)\right\|^{2} \\
& \leq e^{2 \sigma \int_{0}^{t} z\left(\theta_{s-t} \omega\right) d s-F t}\left\|g_{0}\left(\theta_{-t} \omega\right)\right\|^{2} \\
& \quad+c_{1}\|f\|^{2} \int_{0}^{\widetilde{T}} e^{-2 \sigma z\left(\theta_{s-t} \omega\right)+2 \sigma \int_{s}^{t} z\left(\theta_{\tau-t} \omega\right) d \tau+F(s-t)} d s .
\end{aligned}
$$

Apply Gronwall lemma to (4.7) again and denote $d_{0}=\min \left\{d_{1}, d_{2}, d_{3}\right\}, c_{2}=1 / d_{0}$, then for all $t \geq \widetilde{T}$,

$$
\begin{aligned}
\left\|g\left(t, \omega, g_{0}(\omega)\right)\right\|^{2} \leq & e^{2 \sigma \int_{\tilde{T}}^{t} z\left(\theta_{s} \omega\right) d s-F(t-\widetilde{T})}\left\|g\left(\widetilde{T}, \omega, g_{0}(\omega)\right)\right\|^{2} \\
& +c_{1}\|f\|^{2} \int_{\widetilde{T}}^{t} e^{-2 \sigma z\left(\theta_{s} \omega\right)+2 \sigma \int_{s}^{t} z\left(\theta_{\tau} \omega\right) d \tau+F(s-t)} d s \\
& -2 d_{0} \int_{\widetilde{T}}^{t} e^{2 \sigma \int_{s}^{t} z\left(\theta_{\tau} \omega\right) d \tau+F(s-t)}\left\|\nabla g\left(s, \omega, g_{0}(\omega)\right)\right\|^{2} d s,
\end{aligned}
$$

which implies that

$$
\begin{gathered}
\int_{\widetilde{T}}^{t} e^{2 \sigma \int_{s}^{t} z\left(\theta_{\tau} \omega\right) d \tau+F(s-t)}\left\|\nabla g\left(s, \omega, g_{0}(\omega)\right)\right\|^{2} d s \\
\leq \frac{c_{2}}{2} e^{2 \sigma \int_{\tilde{T}}^{t} z\left(\theta_{s} \omega\right) d s-F(t-\widetilde{T})}\left\|g\left(\widetilde{T}, \omega, g_{0}(\omega)\right)\right\|^{2} \\
\quad+\frac{c_{1} c_{2}}{2}\|f\|^{2} \int_{\widetilde{T}}^{t} e^{-2 \sigma z\left(\theta_{s} \omega\right)+2 \sigma \int_{s}^{t} z\left(\theta_{\tau} \omega\right) d \tau+F(s-t)} d s .
\end{gathered}
$$

Replacing $\omega$ in (4.17) with $\theta_{-t} \omega$ and by (4.15), we obtain

$$
\begin{array}{r}
\int_{\widetilde{T}}^{t} e^{2 \sigma \int_{s}^{t} z\left(\theta_{\tau-t} \omega\right) d \tau+F(s-t)}\left\|\nabla g\left(s, \theta_{-t} \omega, g_{0}\left(\theta_{-t} \omega\right)\right)\right\|^{2} d s \\
\leq \frac{c_{2}}{2} e^{2 \sigma \int_{\tilde{T}}^{t} z\left(\theta_{s-t} \omega\right) d s-F(t-\widetilde{T})}\left\|g\left(\widetilde{T}, \theta_{-t} \omega, g_{0}\left(\theta_{-t} \omega\right)\right)\right\|^{2} \\
\quad+\frac{c_{1} c_{2}}{2}\|f\|^{2} \int_{\widetilde{T}}^{t} e^{-2 \sigma z\left(\theta_{s-t} \omega\right)+2 \sigma \int_{s}^{t} z\left(\theta_{\tau-t} \omega\right) d \tau+F(s-t)} d s \\
\leq \frac{c_{2}}{2} e^{2 \sigma \int_{0}^{t} z\left(\theta_{s-t} \omega\right) d s-F t}\left\|g_{0}\left(\theta_{-t} \omega\right)\right\|^{2} \\
\quad+\frac{c_{1} c_{2}}{2}\|f\|^{2} \int_{0}^{t} e^{-2 \sigma z\left(\theta_{s-t} \omega\right)+2 \sigma \int_{s}^{t} z\left(\theta_{\tau-t} \omega\right) d \tau+F(s-t)} d s
\end{array}
$$




$$
\begin{aligned}
= & \frac{c_{2}}{2} e^{2 \sigma \int_{-t}^{0} z\left(\theta_{s} \omega\right) d s-F t}\left\|g_{0}\left(\theta_{-t} \omega\right)\right\|^{2} \\
& +\frac{c_{1} c_{2}}{2}\|f\|^{2} \int_{-t}^{0} e^{-2 \sigma z\left(\theta_{s} \omega\right)+2 \sigma \int_{s}^{0} z\left(\theta_{\tau} \omega\right) d \tau+F s} d s .
\end{aligned}
$$

By substituting $t$ and $\tilde{T}$ for $t+1$ and $t$ in (4.18), we find that

$$
\begin{gathered}
\int_{t}^{t+1} e^{2 \sigma \int_{s}^{t+1} z\left(\theta_{\tau-t-1} \omega\right) d \tau+F(s-t-1)}\left\|\nabla g\left(s, \theta_{-t-1} \omega, g_{0}\left(\theta_{-t-1} \omega\right)\right)\right\|^{2} d s \\
\leq \frac{c_{2}}{2} e^{2 \sigma \int_{-t-1}^{0} z\left(\theta_{s} \omega\right) d s-F(t+1)}\left\|g_{0}\left(\theta_{-t-1} \omega\right)\right\|^{2} \\
+\frac{c_{1} c_{2}}{2}\|f\|^{2} \int_{-t-1}^{0} e^{-2 \sigma z\left(\theta_{s} \omega\right)+2 \sigma \int_{s}^{0} z\left(\theta_{\tau} \omega\right) d \tau+F s} d s .
\end{gathered}
$$

We know for $s \in[t, t+1]$,

$$
\begin{gathered}
\int_{t}^{t+1} e^{2 \sigma \int_{s}^{t+1} z\left(\theta_{\tau-t-1} \omega\right) d \tau+F(s-t-1)}\left\|\nabla g\left(s, \theta_{-t-1} \omega, g_{0}\left(\theta_{-t-1} \omega\right)\right)\right\|^{2} d s \\
\geq \int_{t}^{t+1} e^{-2 \sigma \max _{0 \leq \tau \leq 1}\left|z\left(\theta_{\tau} \omega\right)\right|-F}\left\|\nabla g\left(s, \theta_{-t-1} \omega, g_{0}\left(\theta_{-t-1} \omega\right)\right)\right\|^{2} d s .
\end{gathered}
$$

Due to (3.5) and the temperedness of $g_{0}(\omega)$, there exists a $T_{B}(\omega)>0$ such that for $t \geq T_{B}(\omega)$, and by (4.19) and (4.20), we get

$$
\begin{aligned}
& \int_{t}^{t+1}\left\|\nabla g\left(s, \theta_{-t-1} \omega, g_{0}\left(\theta_{-t-1} \omega\right)\right)\right\|^{2} d s \\
& \leq \frac{c_{2}}{2} e^{2 \sigma \int_{-t-1}^{0} z\left(\theta_{s} \omega\right) d s+2 \sigma \max _{0 \leq \tau \leq 1}\left|z\left(\theta_{\tau} \omega\right)\right|-F t}\left\|g_{0}\left(\theta_{-t-1} \omega\right)\right\|^{2} \\
& \quad+\frac{c_{1} c_{2}}{2}\|f\|^{2} \int_{-t-1}^{0} e^{-2 \sigma z\left(\theta_{s} \omega\right)+2 \sigma \max _{0 \leq \tau \leq 1}\left|z\left(\theta_{\tau} \omega\right)\right|+2 \sigma \int_{s}^{0} z\left(\theta_{\tau} \omega\right) d \tau+F(s+1)} d s \\
& \leq 1+\frac{c_{1} c_{2}}{2}\|f\|^{2} \int_{-\infty}^{0} e^{-2 \sigma z\left(\theta_{s} \omega\right)+2 \sigma \max _{0 \leq \tau \leq 1}\left|z\left(\theta_{\tau} \omega\right)\right|+2 \sigma \int_{s}^{0} z\left(\theta_{\tau} \omega\right) d \tau+F(s+1)} d s \\
& :=\tilde{R}(\omega) .
\end{aligned}
$$

By (3.5) again, we have $\widetilde{R}(\omega)$ is tempered and the proof is completed.

We need the proposition to prove the next result. 
Proposition 4.3. Assume that $f \in \mathbb{H} \cap \mathbb{U}$. Then, there exists a tempered random variable $\widehat{R}(\omega)>0$ such that for any $B(\omega) \in \Phi$ and $g_{0}(\omega) \in B(\omega)$, there exists a $T_{B}(\omega)>0$ such that the solution $\varphi$ of (3.8) satisfies for $\mathbb{P}$-a.e. $\omega \in \Omega$ for all $t \geq T_{B}(\omega)$

$$
\int_{t}^{t+1}\left\|\varphi\left(s, \theta_{-t-1} \omega, g_{0}\left(\theta_{-t-1} \omega\right)\right)\right\|_{\mathbb{U}}^{6} d s \leq \widehat{R}(\omega)
$$

Proof. Let $V(t, x)=v(t, x) / G$, then (4.3)-(4.5) can be written as

$$
\begin{gathered}
\frac{\partial u}{\partial t}=d_{1} \Delta u-\left(F+k-\sigma z\left(\theta_{t} \omega\right)\right) u+\alpha^{-2}(t) u^{2} v-G \alpha^{-2}(t) u^{3}+k W+\alpha(t) f_{1}, \\
\frac{\partial V}{\partial t}=d_{2} \Delta V-\left(F-\sigma z\left(\theta_{t} \omega\right)\right) V-\alpha^{-2}(t) u^{2} V+\alpha^{-2}(t) u^{3}+\frac{1}{G} \alpha(t) f_{2}, \\
\mu \frac{\partial W}{\partial t}=\mu d_{3} \Delta W-\left(\mu F+k-\mu \sigma z\left(\theta_{t} \omega\right)\right) W+k u+\alpha(t) f_{3} .
\end{gathered}
$$

Taking the inner products of (4.23) with $u^{5}(t), G V^{5}(t)$, and $W^{5}(t)$, and summing up the resulting equalities, we get

$$
\begin{aligned}
\frac{1}{6} \frac{d}{d t}( & \left.\|u\|_{L^{6}}^{6}+G\|V\|_{L^{6}}^{6}+\mu\|W\|_{L^{6}}^{6}\right)+5\left(d_{1}\left\|u^{2} \nabla u\right\|^{2}+d_{2} G\left\|V^{2} \nabla V\right\|^{2}+\mu d_{3} G\left\|W^{2} \nabla W\right\|^{2}\right) \\
= & -\left(F+k-\sigma z\left(\theta_{t} \omega\right)\right)\|u\|_{L^{6}}^{6}-G\left(F-\sigma z\left(\theta_{t} \omega\right)\right)\|V\|_{L^{6}}^{6} \\
& -\left(\mu F+k-\mu \sigma z\left(\theta_{t} \omega\right)\right)\|W\|_{L^{6}}^{6}+k \int_{\mathbb{R}^{n}} u^{5} W d x+k \int_{\mathbb{R}^{n}} u W^{5} d x \\
& -G \alpha^{-2}(t) \int_{\mathbb{R}^{n}}\left(u^{8}-u^{7} V-u^{3} V^{5}+u^{2} V^{6}\right) d x \\
& +\int_{\mathbb{R}^{n}} \alpha(t) f_{1} u^{5} d x+\int_{\mathbb{R}^{n}} \alpha(t) f_{2} V^{5} d x+\int_{\mathbb{R}^{n}} \alpha(t) f_{3} W^{5} d x,
\end{aligned}
$$

that is,

$$
\begin{aligned}
\frac{d}{d t}\left(\|u\|_{L^{6}}^{6}+G\|V\|_{L^{6}}^{6}+\mu\|W\|_{L^{6}}^{6}\right) \\
\leq-6\left(F-\sigma z\left(\theta_{t} \omega\right)\right)\|u\|_{L^{6}}^{6}-6 G\left(F-\sigma z\left(\theta_{t} \omega\right)\right)\|V\|_{L^{6}}^{6}-6\left(\mu F-\mu \sigma z\left(\theta_{t} \omega\right)\right)\|W\|_{L^{6}}^{6} \\
\quad+6 \int_{\mathbb{R}^{n}} \alpha(t) f_{1} u^{5} d x+6 \int_{\mathbb{R}^{n}} \alpha(t) f_{2} V^{5} d x+6 \int_{\mathbb{R}^{n}} \alpha(t) f_{3} W^{5} d x \\
\leq\left(6 \sigma z\left(\theta_{t} \omega\right)-F\right)\|u\|_{L^{6}}^{6}+G\left(6 \sigma z\left(\theta_{t} \omega\right)-F\right)\|V\|_{L^{6}}^{6}+\mu\left(6 \sigma z\left(\theta_{t} \omega\right)-F\right)\|W\|_{L^{6}}^{6} \\
\quad+\frac{1}{F^{5}} \alpha^{6}(t)\left(\left\|f_{1}\right\|_{L^{6}}^{6}+\frac{1}{G^{5}}\left\|f_{2}\right\|_{L^{6}}^{6}+\frac{1}{\mu^{5}}\left\|f_{3}\right\|_{L^{6}}^{6}\right) .
\end{aligned}
$$


Furthermore, let

$$
c_{3}=\frac{\max \left\{1,1 / G^{5}, 1 / \mu^{5}\right\}}{F^{5} \min \left\{1,1 / G^{5}, 1 / \mu^{5}\right\}}
$$

and apply Gronwall lemma, for $v \geq 0$, we can deduce from (4.25) that

$$
\begin{aligned}
& \left\|g\left(v, \omega, g_{0}(\omega)\right)\right\|_{\mathbb{U}}^{6} \leq e^{6 \sigma \int_{0}^{v} z\left(\theta_{s} \omega\right) d s-F v}\left\|g_{0}(\omega)\right\|_{\mathbb{U}}^{6} \\
& \quad+c_{3}\|f\|_{\mathbb{U}}^{6} e^{6 \sigma \int_{0}^{v} z\left(\theta_{s} \omega\right) d s-F v} \int_{0}^{v} e^{-6 \sigma z\left(\theta_{s} \omega\right)-6 \sigma \int_{0}^{s} z\left(\theta_{\tau} \omega\right) d \tau+F s} d s .
\end{aligned}
$$

Letting $T_{B}(\omega)$ be a positive variable in Lemma 4.2, $t \geq T_{B}(\omega)$, integrating (4.27) for $v \in(t, t+1)$, we have

$$
\begin{aligned}
\int_{t}^{t+1} & \left\|g\left(v, \omega, g_{0}(\omega)\right)\right\|_{\mathbb{U}}^{6} d T \leq e^{6 \sigma \int_{0}^{t+1} z\left(\theta_{s} \omega\right) d s-F t}\left\|g_{0}(\omega)\right\|_{\mathbb{U}}^{6} \\
& +c_{3}\|f\|_{\mathbb{U}}^{6} e^{6 \sigma \int_{0}^{t+1} z\left(\theta_{s} \omega\right) d s-F t} \int_{0}^{t+1} e^{-6 \sigma z\left(\theta_{s} \omega\right)-6 \sigma \int_{0}^{s} z\left(\theta_{\tau} \omega\right) d \tau+F s} d s
\end{aligned}
$$

Replacing $\omega$ by $\theta_{-t-1} \omega$ in (4.28), we obtain

$$
\begin{aligned}
\int_{t}^{t+1} & \left\|g\left(v, \theta_{-t-1} \omega, g_{0}\left(\theta_{-t-1} \omega\right)\right)\right\|_{\mathbb{U}}^{6} d v \\
\leq & e^{6 \sigma \int_{0}^{t+1} z\left(\theta_{s-t-1} \omega\right) d s-F t}\left\|g_{0}\left(\theta_{-t-1} \omega\right)\right\|_{\mathbb{U}}^{6} \\
& +c_{3}\|f\|_{\mathbb{U}}^{6} e^{6 \sigma \int_{0}^{t+1} z\left(\theta_{s-t-1} \omega\right) d s-F t} \int_{0}^{t+1} e^{-6 \sigma z\left(\theta_{s-t-1} \omega\right)-6 \sigma \int_{0}^{s} z\left(\theta_{\tau-t-1} \omega\right) d \tau+F s} d s \\
\leq & e^{6 \sigma \int_{-t-1}^{0} z\left(\theta_{s} \omega\right) d s-F t}\left\|g_{0}\left(\theta_{-t-1} \omega\right)\right\|_{\mathbb{U}}^{6} \\
& +c_{3}\|f\|_{\mathbb{U}}^{6} e^{6 \sigma \int_{-t-1}^{0} z\left(\theta_{s} \omega\right) d s-F t} \int_{-t-1}^{0} e^{-6 \sigma z\left(\theta_{s} \omega\right)-6 \sigma \int_{s}^{0} z\left(\theta_{\tau} \omega\right) d \tau+F s} d s \\
\leq & 1+c_{3}\|f\|_{\mathbb{U}}^{6} \int_{-\infty}^{0} e^{-6 \sigma z\left(\theta_{s} \omega\right)-6 \sigma \int_{s}^{0} z\left(\theta_{\tau} \omega\right) d \tau+F s} d s \\
:= & \widehat{R}(\omega) .
\end{aligned}
$$

The last but one line in (4.29) due to $B(\omega) \in \Phi$ is tempered, then for any $g_{0}\left(\theta_{-t-1} \omega\right) \in$ $B\left(\theta_{-t-1} \omega\right) \subset B\left(\theta_{-t} \omega\right)$,

$$
\lim _{t \rightarrow+\infty} e^{6 \sigma \int_{-t-1}^{0} z\left(\theta_{s} \omega\right) d s-F t}\left\|g_{0}\left(\theta_{-t-1} \omega\right)\right\|_{\mathbb{U}}^{6}=0
$$

The proof is completed. 
Lemma 4.4. Assume that $f \in \mathbb{H} \cap \mathbb{U}$. Then, there exists a tempered random variable $\tilde{R}(\omega)>0$ such that for any $B(\omega) \in \Phi$ and $g_{0}(\omega) \in B(\omega)$, there exists a $T_{B}(\omega)>0$ such that the solution $\varphi$ of (3.8) satisfies for $\mathbb{P}$-a.e. $\omega \in \Omega$, for all $t \geq T_{B}(\omega)$,

$$
\left\|\nabla \varphi\left(t, \theta_{-t} \omega, g_{0}\left(\theta_{-t} \omega\right)\right)\right\|^{2} \leq R(\omega)
$$

Proof. Taking the inner products of (4.3)-(4.5) with $-\Delta u,-\Delta v,-\Delta W$, respectively, and summing up the three resulting equalities, we have

$$
\begin{aligned}
\frac{1}{2} \frac{d}{d t}( & \left.\|\nabla u\|^{2}+\|\nabla v\|^{2}+\mu\|\nabla W\|^{2}\right)+d_{1}\|\Delta u\|^{2}+d_{2}\|\Delta v\|^{2}+\mu d_{3}\|\Delta W\|^{2} \\
& +\left(F+k-\sigma z\left(\theta_{t} \omega\right)\right)\|\nabla u\|^{2}+\left(F-\sigma z\left(\theta_{t} \omega\right)\right)\|\nabla v\|^{2} \\
& +\left(\mu F+k-\mu \sigma z\left(\theta_{t} \omega\right)\right)\|\nabla W\|^{2} \\
= & -\alpha^{-2}(t) \int_{\mathbb{R}^{n}} u^{2} v \Delta u d x+G \alpha^{-2}(t) \int_{\mathbb{R}^{n}} u^{3} \Delta u d x \\
& +\alpha^{-2}(t) \int_{\mathbb{R}^{n}} u^{2} v \Delta v d x-G \alpha^{-2}(t) \int_{\mathbb{R}^{n}} u^{3} \Delta v d x-2 k \int_{\mathbb{R}^{n}} u \Delta W d x \\
& -\int_{\mathbb{R}^{n}} \alpha(t) \Delta u f_{1} d x-\int_{\mathbb{R}^{n}} \alpha(t) \Delta v f_{2} d x-\int_{\mathbb{R}^{n}} \alpha(t) \Delta W f_{3} d x .
\end{aligned}
$$

By Hölder inequality, it yields

$$
\begin{aligned}
\frac{d}{d t}( & \left.\|\nabla u\|^{2}+\|\nabla v\|^{2}+\mu\|\nabla W\|^{2}\right) \\
& +\left(F-2 \sigma z\left(\theta_{t} \omega\right)\right)\left(\|\nabla u\|^{2}+\|\nabla v\|^{2}+\mu\|\nabla W\|^{2}\right) \\
\leq & \left(\frac{2}{d_{1}}+\frac{2}{d_{2}}\right) \alpha^{-4}(t) \int_{\mathbb{R}^{n}} u^{4} v^{2} d x+\left(\frac{2}{d_{1}}+\frac{2}{d_{2}}\right) G^{2} \alpha^{-4}(t) \int_{\mathbb{R}^{n}} u^{6} d x \\
& +\frac{1}{d_{1}} \alpha^{2}(t)\left\|f_{1}\right\|^{2}+\frac{1}{d_{2}} \alpha^{2}(t)\left\|f_{2}\right\|^{2}+\frac{1}{\mu d_{3}} \alpha^{2}(t)\left\|f_{3}\right\|^{2} \\
\leq & \frac{1}{d_{0}}\left(\frac{8}{3}+4 G^{2}\right) \alpha^{-4}(t)\left(\|u\|_{L^{6}}^{6}+\|v\|_{L^{6}}^{6}\right)+\frac{1}{d_{0}} \alpha^{2}(t)\|f\|^{2}
\end{aligned}
$$

which implies that

$$
\frac{d}{d t}\|\nabla g\|^{2} \leq\left(2 \sigma z\left(\theta_{t} \omega\right)-F\right)\|\nabla g\|^{2}+c_{4} \alpha^{-4}(t)\|g\|_{\mathbb{U}}^{6}+c_{5} \alpha^{2}(t)\|f\|^{2}
$$

where $c_{4}=c_{2}\left(8 / 3+4 G^{2}\right) / \min \{1,1 / \mu\}$ and $c_{5}=c_{2} / \min \{1,1 / \mu\}$. 
Let $T_{B}(\omega)$ be a positive variable in Lemma $4.2, t \geq T_{B}(\omega)$ and $s \in(t, t+1)$. Then integrate (4.34) over $(s, t+1)$, we get

$$
\begin{aligned}
& \left\|\nabla g\left(t+1, \omega, g_{0}(\omega)\right)\right\|^{2} \\
& \leq\left\|\nabla g\left(s, \omega, g_{0}(\omega)\right)\right\|^{2}+\int_{s}^{t+1}\left(2 \sigma z\left(\theta_{\tau} \omega\right)-F\right)\left\|\nabla g\left(\tau, \omega, g_{0}(\omega)\right)\right\|^{2} d \tau \\
& \quad+c_{4} \int_{s}^{t+1} \alpha^{-4}(\tau)\left\|g\left(\tau, \omega, g_{0}(\omega)\right)\right\|_{\mathbb{U}}^{6} d \tau \\
& \quad+c_{5} \int_{s}^{t+1} \alpha^{2}(\tau)\|f\|^{2} d \tau .
\end{aligned}
$$

Now integrating (4.35) with respect to $s$ over $(t, t+1)$, we find that

$$
\begin{aligned}
\left\|\nabla g\left(t+1, \omega, g_{0}(\omega)\right)\right\|^{2} \leq & (1-F) \int_{t}^{t+1}\left\|\nabla g\left(\tau, \omega, g_{0}(\omega)\right)\right\|^{2} d \tau \\
& +2 \sigma \int_{t}^{t+1} z\left(\theta_{\tau} \omega\right)\left\|\nabla g\left(\tau, \omega, g_{0}(\omega)\right)\right\|^{2} d \tau \\
& +c_{4} \int_{t}^{t+1} \alpha^{-4}(\tau)\left\|g\left(\tau, \omega, g_{0}(\omega)\right)\right\|_{\mathbb{U}}^{6} d \tau \\
& +c_{5} \int_{t}^{t+1} \alpha^{2}(\tau)\|f\|^{2} d \tau .
\end{aligned}
$$

Since $z(\omega)$ is tempered and $z\left(\theta_{t} \omega\right)$ is continuous in $t$, there exists a tempered variable $r(\omega)$ such that

$$
\left|z\left(\theta_{t} \omega\right)\right| \leq e^{|t|} r(\omega), \quad \forall t \in \mathbb{R}^{n}
$$

Then, replacing $\omega$ with $\theta_{-t-1} \omega$, by Lemma 4.2 and Proposition 4.3, it follows in (4.36) that

$$
\begin{aligned}
& \left\|\nabla g\left(t+1, \theta_{-t-1} \omega, g_{0}\left(\theta_{-t-1} \omega\right)\right)\right\|^{2} \\
& \leq(1-F) \int_{t}^{t+1}\left\|\nabla g\left(\tau, \theta_{-t-1} \omega, g_{0}\left(\theta_{-t-1} \omega\right)\right)\right\|^{2} d \tau \\
& \quad+2 \sigma \int_{t}^{t+1} z\left(\theta_{\tau-t-1} \omega\right)\left\|\nabla g\left(\tau, \theta_{-t-1} \omega, g_{0}\left(\theta_{-t-1} \omega\right)\right)\right\|^{2} d \tau \\
& \quad+c_{4} \int_{t}^{t+1} e^{4 \sigma z\left(\theta_{\tau-t-1} \omega\right)}\left\|g\left(\tau, \theta_{-t-1} \omega, g_{0}\left(\theta_{-t-1} \omega\right)\right)\right\|_{\mathbb{U}}^{6} d \tau \\
& \quad+c_{5} \int_{t}^{t+1} e^{-2 \sigma z\left(\theta_{\tau-t-1} \omega\right)}\|f\|^{2} d \tau
\end{aligned}
$$


Abstract and Applied Analysis

$$
\begin{aligned}
& \leq(1-F+2 e \sigma r(\omega)) \int_{t}^{t+1}\left\|\nabla g\left(\tau, \theta_{-t-1} \omega, g_{0}\left(\theta_{-t-1} \omega\right)\right)\right\|^{2} d \tau
\end{aligned}
$$

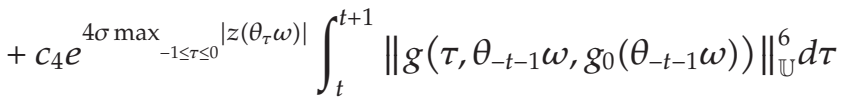

$$
\begin{aligned}
& +c_{5} \int_{-1}^{0} e^{-2 \sigma z\left(\theta_{\tau} \omega\right)}\|f\|^{2} d \tau
\end{aligned}
$$

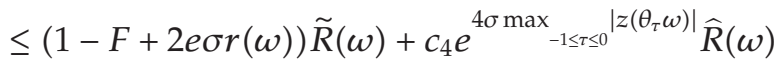

$$
\begin{aligned}
& +c_{5} \int_{-1}^{0} e^{-2 \sigma z\left(\theta_{\tau} \omega\right)}\|f\|^{2} d \tau \\
& :=R(\omega) \text {. }
\end{aligned}
$$

Note that $f \in \mathbb{H} \cap \mathbb{U}$. It is easy to see that $R(\omega)$ is tempered. This completes the proof.

Lemma 4.5. Assume that $f \in \mathbb{H}$. Let $\{B(\omega)\} \in \Phi$ and $g_{0}(\omega) \in B(\omega)$. Then, for every $\epsilon>0$, there exist $T^{*}=T(\epsilon, \omega, B)>0$ and $K^{*}=K(\epsilon, \omega)>0$ such that the solution $\varphi$ of problem (3.8) satisfies for $\mathbb{P}$-a.e. $\omega \in \Omega$, for all $t \geq T$,

$$
\int_{|x| \geq K^{*}}\left|\varphi\left(t, \theta_{-t} \omega, g_{0}\left(\theta_{-t} \omega\right)\right)\right|^{2} d x \leq \epsilon
$$

Proof. Choose a smooth cutoff function satisfying $0 \leq \rho(s) \leq 1$ for $s \in \mathbb{R}^{+}$and $\rho(s)=0$ for $0 \leq s \leq 1, \rho(s)=1$ for $s \geq 2$. Suppose there exists a constant $c$ such that $\left|\rho^{\prime}(s)\right| \leq c$ for $s \in \mathbb{R}^{+}$.

Taking the inner product of (4.3), (4.4), and (4.5) with $G \rho\left(|x|^{2} / K^{2}\right) u, \rho\left(|x|^{2} / K^{2}\right) v$ and $G \rho\left(|x|^{2} / K^{2}\right) W$ in $L^{2}\left(\mathbb{R}^{n}\right)$, respectively, we get

$$
\begin{aligned}
& \frac{G}{2} \frac{d}{d t} \int_{\mathbb{R}^{n}} \rho\left(\frac{|x|^{2}}{K^{2}}\right)|u|^{2} d x-d_{1} G \int_{\mathbb{R}^{n}} \rho\left(\frac{|x|^{2}}{K^{2}}\right) u \Delta u d x \\
& +G\left(F+k-\sigma z\left(\theta_{t} \omega\right)\right) \int_{\mathbb{R}^{n}} \rho\left(\frac{|x|^{2}}{K^{2}}\right)|u|^{2} d x \\
& =G \int_{\mathbb{R}^{n}} \rho\left(\frac{|x|^{2}}{K^{2}}\right) \alpha^{-2}(t) u^{3} v d x-G^{2} \int_{\mathbb{R}^{n}} \rho\left(\frac{|x|^{2}}{K^{2}}\right) \alpha^{-2}(t) u^{4} d x \\
& +k G \int_{\mathbb{R}^{n}} \rho\left(\frac{|x|^{2}}{K^{2}}\right) u W d x+G \int_{\mathbb{R}^{n}} \rho\left(\frac{|x|^{2}}{K^{2}}\right) \alpha(t) f_{1} u d x,
\end{aligned}
$$




$$
\begin{aligned}
& \frac{1}{2} \frac{d}{d t} \int_{\mathbb{R}^{n}} \rho\left(\frac{|x|^{2}}{K^{2}}\right)|v|^{2} d x-d_{2} \int_{\mathbb{R}^{n}} \rho\left(\frac{|x|^{2}}{K^{2}}\right) v \Delta v d x+\left(F-\sigma z\left(\theta_{t} \omega\right)\right) \int_{\mathbb{R}^{n}} \rho\left(\frac{|x|^{2}}{K^{2}}\right)|v|^{2} d x \\
& =-\int_{\mathbb{R}^{n}} \rho\left(\frac{|x|^{2}}{K^{2}}\right) \alpha^{-2}(t) u^{2} v^{2} d x+G \int_{\mathbb{R}^{n}} \rho\left(\frac{|x|^{2}}{K^{2}}\right) \alpha^{-2}(t) v u^{3} d x \\
& \quad+\int_{\mathbb{R}^{n}} \rho\left(\frac{|x|^{2}}{K^{2}}\right) \alpha(t) f_{2} v d x, \\
& \frac{\mu G}{2} \frac{d}{d t} \int_{\mathbb{R}^{n}} \rho\left(\frac{|x|^{2}}{K^{2}}\right)|W|^{2} d x-\mu G d_{3} \int_{\mathbb{R}^{n}} \rho\left(\frac{|x|^{2}}{K^{2}}\right) W \Delta W d x \\
& \quad+G\left(\mu F+k-\sigma z\left(\theta_{t} \omega\right)\right) \int_{\mathbb{R}^{n}} \rho\left(\frac{|x|^{2}}{K^{2}}\right)|W|^{2} d x \\
& =k G \int_{\mathbb{R}^{n}} \rho\left(\frac{|x|^{2}}{K^{2}}\right) W u d x+G \int_{\mathbb{R}^{n}} \rho\left(\frac{|x|^{2}}{K^{2}}\right) \alpha(t) f_{3} W d x .
\end{aligned}
$$

Adding up the three equalities, we have

$$
\begin{aligned}
& \frac{d}{d t}\left(G \int_{\mathbb{R}^{n}} \rho\left(\frac{|x|^{2}}{K^{2}}\right)|u|^{2} d x+\int_{\mathbb{R}^{n}} \rho\left(\frac{|x|^{2}}{K^{2}}\right)|v|^{2} d x+\mu G \int_{\mathbb{R}^{n}} \rho\left(\frac{|x|^{2}}{K^{2}}\right)|W|^{2} d x\right) \\
& \quad-2\left(d_{1} G \int_{\mathbb{R}^{n}} \rho\left(\frac{|x|^{2}}{K^{2}}\right) u \Delta u d x+d_{2} \int_{\mathbb{R}^{n}} \rho\left(\frac{|x|^{2}}{K^{2}}\right) v \Delta v d x+\mu G d_{3} \int_{\mathbb{R}^{n}} \rho\left(\frac{|x|^{2}}{K^{2}}\right) W \Delta W d x\right) \\
& \quad+G\left(F-2 \sigma z\left(\theta_{t} \omega\right)\right) \int_{\mathbb{R}^{n}} \rho\left(\frac{|x|^{2}}{K^{2}}\right)|u|^{2} d x \\
& \quad+\left(F-2 \sigma z\left(\theta_{t} \omega\right)\right) \int_{\mathbb{R}^{n}} \rho\left(\frac{|x|^{2}}{K^{2}}\right)|v|^{2} d x+G\left(\mu F-2 \sigma z\left(\theta_{t} \omega\right)\right) \int_{\mathbb{R}^{n}} \rho\left(\frac{|x|^{2}}{K^{2}}\right)|W|^{2} d x \\
& \leq \frac{G}{2 F} \alpha^{2}(t) \int_{\mathbb{R}^{n}} \rho\left(\frac{|x|^{2}}{K^{2}}\right)\left|f_{1}(x, t)\right|^{2} d x+\frac{G}{2 \mu F} \alpha^{2}(t) \int_{\mathbb{R}^{n}} \rho\left(\frac{|x|^{2}}{K^{2}}\right)\left|f_{3}(x, t)\right|^{2} d x \\
& \quad+\frac{1}{2 F} \int_{\mathbb{R}^{n}} \alpha^{2}(t) \rho\left(\frac{|x|^{2}}{K^{2}}\right)\left|f_{2}(x, t)\right|^{2} d x .
\end{aligned}
$$

We know that

$$
\int_{\mathbb{R}^{n}} \rho\left(\frac{|x|^{2}}{K^{2}}\right) u \Delta u d x \leq-\int_{\mathbb{R}^{n}} \rho\left(\frac{|x|^{2}}{K^{2}}\right)|\nabla u|^{2} d x+\frac{c_{6}}{K}\left(\|u\|^{2}+\|\nabla u\|^{2}\right),
$$


where $c_{6}$ is a positive constant which depends on $c$. Then from (4.41) and (4.42), we get

$$
\begin{aligned}
& \frac{d}{d t}\left(G \int_{\mathbb{R}^{n}} \rho\left(\frac{|x|^{2}}{K^{2}}\right)|u|^{2} d x+\int_{\mathbb{R}^{n}} \rho\left(\frac{|x|^{2}}{K^{2}}\right)|v|^{2} d x+\mu G \int_{\mathbb{R}^{n}} \rho\left(\frac{|x|^{2}}{K^{2}}\right)|W|^{2} d x\right) \\
& +G\left(F-2 \sigma z\left(\theta_{t} \omega\right)\right) \int_{\mathbb{R}^{n}} \rho\left(\frac{|x|^{2}}{K^{2}}\right)|u|^{2} d x+\left(F-2 \sigma z\left(\theta_{t} \omega\right)\right) \int_{\mathbb{R}^{n}} \rho\left(\frac{|x|^{2}}{K^{2}}\right)|v|^{2} d x \\
& +G\left(\mu F-2 \sigma z\left(\theta_{t} \omega\right)\right) \int_{\mathbb{R}^{n}} \rho\left(\frac{|x|^{2}}{K^{2}}\right)|W|^{2} d x \\
& \leq \frac{G}{2 F} \alpha^{2}(t) \int_{\mathbb{R}^{n}} \rho\left(\frac{|x|^{2}}{K^{2}}\right)\left|f_{1}(x, t)\right|^{2} d x+\frac{G}{2 \mu F} \alpha^{2}(t) \int_{\mathbb{R}^{n}} \rho\left(\frac{|x|^{2}}{K^{2}}\right)\left|f_{3}(x, t)\right|^{2} d x \\
& \quad+\frac{1}{2 F} \int_{\mathbb{R}^{n}} \alpha^{2}(t) \rho\left(\frac{|x|^{2}}{K^{2}}\right)\left|f_{2}(x, t)\right|^{2} d x+\frac{2 d^{0} c_{6}}{K}\left(G\|u\|^{2}+\|v\|^{2}+\mu G\|W\|^{2}\right) \\
& +\frac{2 d^{0} c_{6}}{K}\left(G\|\nabla u\|^{2}+\|\nabla v\|^{2}+\mu G\|\nabla W\|^{2}\right) .
\end{aligned}
$$

Defining

$$
c_{7}=\frac{\max \{1, G, G / \mu\}}{\min \{1, G, G / \mu\}}, \quad c_{8}=2 d^{0} c_{6} c_{7}, \quad d^{0}=\max \left\{d_{1}, d_{2}, d_{3}\right\}
$$

and by Gronwall lemma, for any $t \geq \tilde{T}$, we obtain

$$
\begin{aligned}
\int_{\mathbb{R}^{n}} \rho\left(\frac{|x|^{2}}{K^{2}}\right)\left|g\left(t, \omega, g_{0}(\omega)\right)\right|^{2} d x \\
\leq e^{2 \sigma \int_{\widetilde{T}}^{t} z\left(\theta_{\tau} \omega\right) d \tau-F(t-\widetilde{T})} \int_{\mathbb{R}^{n}} \rho\left(\frac{|x|^{2}}{K^{2}}\right)\left|g\left(\widetilde{T}, \omega, g_{0}(\omega)\right)\right|^{2} d x \\
\quad+c_{7} \int_{\widetilde{T}}^{t} e^{2 \sigma \int_{s}^{t} z\left(\theta_{\tau} \omega\right) d \tau-F(t-s)-2 \sigma z\left(\theta_{s} \omega\right)} \int_{\mathbb{R}^{n}} \rho\left(\frac{|x|^{2}}{K^{2}}\right) f^{2} d x d s \\
\quad+\frac{c_{8}}{K} \int_{\widetilde{T}}^{t} e^{2 \sigma \int_{s}^{t} z\left(\theta_{\tau} \omega\right) d \tau-F(t-s)}\left\|g\left(s, \omega, g_{0}(\omega)\right)\right\|^{2} d s \\
\quad+\frac{c_{8}}{K} \int_{\widetilde{T}}^{t} e^{2 \sigma \int_{s}^{t} z\left(\theta_{\tau} \omega\right) d \tau-F(t-s)}\left\|\nabla g\left(s, \omega, g_{0}(\omega)\right)\right\|^{2} d s .
\end{aligned}
$$


By replacing $\omega$ by $\theta_{-t} \omega$, it then follows from (4.45) that

$$
\begin{aligned}
& \int_{\mathbb{R}^{n}} \rho\left(\frac{|x|^{2}}{K^{2}}\right)\left|g\left(t, \theta_{-t} \omega, g_{0}\left(\theta_{-t} \omega\right)\right)\right|^{2} d x \\
& \leq e^{2 \sigma \int_{\tilde{T}}^{t} z\left(\theta_{\tau-t} \omega\right) d \tau-F(t-\tilde{T})} \int_{\mathbb{R}^{n}} \rho\left(\frac{|x|^{2}}{K^{2}}\right)\left|g\left(\widetilde{T}, \theta_{-t} \omega, g_{0}\left(\theta_{-t} \omega\right)\right)\right|^{2} d x \\
& \quad+c_{7} \int_{\widetilde{T}}^{t} e^{2 \sigma \int_{s}^{t} z\left(\theta_{\tau-t} \omega\right) d \tau-F(t-s)-2 \sigma z\left(\theta_{s-t} \omega\right)} \int_{\mathbb{R}^{n}} \rho\left(\frac{|x|^{2}}{K^{2}}\right) f^{2} d x d s \\
& \quad+\frac{c_{8}}{K} \int_{\widetilde{T}}^{t} e^{2 \sigma \int_{s}^{t} z\left(\theta_{\tau-t} \omega\right) d \tau-F(t-s)}\left\|g\left(s, \theta_{-t} \omega, g_{0}\left(\theta_{-t} \omega\right)\right)\right\|^{2} d s \\
& \quad+\frac{c_{8}}{K} \int_{\widetilde{T}}^{t} e^{2 \sigma \int_{s}^{t} z\left(\theta_{\tau-t} \omega\right) d \tau-F(t-s)}\left\|\nabla g\left(s, \theta_{-t} \omega, g_{0}\left(\theta_{-t} \omega\right)\right)\right\|^{2} d s .
\end{aligned}
$$

We now estimate each term in (4.46) on the right-hand side one by one. By substituting $t$ by $\tilde{T}$ and $\omega$ by $\theta_{-t} \omega$ in (4.8) and combine with the first term of (4.46), we get

$$
\begin{aligned}
& e^{2 \sigma \int_{\tilde{T}}^{t} z\left(\theta_{\tau-t} \omega\right) d \tau-F(t-\widetilde{T})} \int_{\mathbb{R}^{n}} \rho\left(\frac{|x|^{2}}{K^{2}}\right)\left|g\left(\widetilde{T}, \theta_{-t} \omega, g_{0}\left(\theta_{-t} \omega\right)\right)\right|^{2} d x \\
& \leq e^{2 \sigma \int_{\tilde{T}}^{t} z\left(\theta_{\tau-t} \omega\right) d \tau-F(t-\widetilde{T})}\left(e^{2 \sigma \int_{0}^{\tilde{T}} z\left(\theta_{s-t} \omega\right) d s-F \tilde{T}}\left\|g_{0}\left(\theta_{-t} \omega\right)\right\|^{2}\right. \\
& \left.+c_{1}\|f\|^{2} e^{2 \sigma \int_{0}^{\tilde{T}} z\left(\theta_{s-t} \omega\right) d s-F \tilde{T}} \int_{0}^{\tilde{T}} e^{-2 \sigma z\left(\theta_{s-t} \omega\right)-2 \sigma \int_{0}^{s} z\left(\theta_{\tau-t} \omega\right) d \tau+F s} d s\right) \\
& =e^{2 \sigma \int_{0}^{t} z\left(\theta_{\tau-t} \omega\right) d \tau-F t}\left\|g_{0}\left(\theta_{-t} \omega\right)\right\|^{2} \\
& +c_{1}\|f\|^{2} \int_{0}^{\tilde{T}} e^{-2 \sigma z\left(\theta_{s-t} \omega\right)+2 \sigma \int_{s}^{t} z\left(\theta_{\tau-t} \omega\right) d \tau-F(t-s)} d s .
\end{aligned}
$$

Obviously, there exists $T_{1}=T_{1}(\epsilon, \omega, B)>\widetilde{T}$ such that for $t>T_{1}$,

$$
e^{2 \sigma \int_{\widetilde{T}}^{t} z\left(\theta_{\tau-t} \omega\right) d \tau-F(t-\widetilde{T})} \int_{\mathbb{R}^{n}} \rho\left(\frac{|x|^{2}}{K^{2}}\right)\left|g\left(\widetilde{T}, \theta_{-t} \omega, g_{0}\left(\theta_{-t} \omega\right)\right)\right|^{2} d x \leq \epsilon
$$


For the second term on the right-hand side of (4.46), since $f \in \mathbb{H}$, there exist $T_{2}=T_{2}(\epsilon, \omega, B)>$ $\tilde{T}$ and $K_{1}=K_{1}(\epsilon, \omega)>0$ such that for all $t>T_{2}$ and $K>K_{1}$, then

$$
\begin{aligned}
& c_{7} \int_{\widetilde{T}}^{t} e^{2 \sigma \int_{s}^{t} z\left(\theta_{\tau-t} \omega\right) d \tau-F(t-s)-2 \sigma z\left(\theta_{s-t} \omega\right)} \int_{\mathbb{R}^{n}} \rho\left(\frac{|x|^{2}}{K^{2}}\right) f^{2} d x d s \\
& \quad \leq c_{7} \int_{\widetilde{T}}^{t} e^{2 \sigma \int_{s}^{t} z\left(\theta_{\tau-t} \omega\right) d \tau-F(t-s)-2 \sigma z\left(\theta_{s-t} \omega\right)} \int_{|x| \geq K} \rho\left(\frac{|x|^{2}}{K^{2}}\right) f^{2} d x d s
\end{aligned}
$$

$\leq \epsilon$.

For the third term, by replacing $t$ by $s$ and $\omega$ by $\theta_{-t} \omega$ in (4.8)

$$
\begin{aligned}
& \frac{c_{8}}{K} \int_{\widetilde{T}}^{t} e^{2 \sigma \int_{s}^{t} z\left(\theta_{\tau-t} \omega\right) d \tau-F(t-s)}\left\|g\left(s, \theta_{-t} \omega, g_{0}\left(\theta_{-t} \omega\right)\right)\right\|^{2} d s \\
& \leq \frac{c_{8}}{K} \int_{\widetilde{T}}^{t} e^{2 \sigma t_{s}^{t} z\left(\theta_{\tau-t} \omega\right) d \tau-F(t-s)}\left(e^{2 \sigma \int_{0}^{s} z\left(\theta_{s-t} \omega\right) d s-F s}\left\|g_{0}\left(\theta_{-t} \omega\right)\right\|^{2}\right. \\
& \left.\quad+c_{1}\|f\|^{2} e^{2 \sigma \int_{0}^{s} z\left(\theta_{s-t} \omega\right) d s-F s} \int_{0}^{s} e^{-2 \sigma z\left(\theta_{v-t} \omega\right)-2 \sigma \int_{0}^{v} z\left(\theta_{\tau-t} \omega\right) d \tau+F v} d v\right) d s \\
& \leq \frac{c_{8}}{K}(t-\tilde{T}) e^{2 \sigma \int_{0}^{t} z\left(\theta_{s-t} \omega\right) d s-F t}\left\|g_{0}\left(\theta_{-t} \omega\right)\right\|^{2} \\
& \quad+\frac{c_{8} c_{1}\|f\|^{2}}{K} \int_{\widetilde{T}}^{t} \int_{0}^{s} e^{-2 \sigma z\left(\theta_{v-t} \omega\right)+2 \sigma \int_{v}^{t} z\left(\theta_{\tau-t} \omega\right) d \tau-F(t-v)} d v d s .
\end{aligned}
$$

Since $f \in \mathbb{H}$, there exist $T_{3}=T_{3}(\epsilon, \omega, B)>\widetilde{T}$ and $K_{2}=K_{2}(\epsilon, \omega)>0$ such that for all $t>T_{3}$ and $K>K_{2}$, we have

$$
\frac{c_{8}}{K} \int_{\widetilde{T}}^{t} e^{2 \sigma \int_{s}^{t} z\left(\theta_{\tau-t} \omega\right) d \tau-F(t-s)}\left\|g\left(s, \theta_{-t} \omega, g_{0}\left(\theta_{-t} \omega\right)\right)\right\|^{2} d s \leq \epsilon
$$

Finally, we estimate the last term on the right-hand side of (4.46). Since $f \in \mathbb{H}$, by using (4.18), there exist $T_{4}=T_{4}(\epsilon, \omega, B)>\widetilde{T}$ and $K_{3}=K_{3}(\epsilon, \omega)>0$ such that for all $t>T_{4}$ and $K>K_{3}$, we obtain

$$
\frac{c_{8}}{K} \int_{\widetilde{T}}^{t} e^{2 \sigma \int_{s}^{t} z\left(\theta_{\tau-t} \omega\right) d \tau-F(t-s)}\left\|\nabla g\left(s, \theta_{-t} \omega, g_{0}\left(\theta_{-t} \omega\right)\right)\right\|^{2} d s \leq \epsilon
$$

Now, denoting

$$
T^{*}=\max \left\{T_{1}, T_{2}, T_{3}, T_{4}\right\}, \quad K^{*}=\max \left\{K_{1}, K_{2}, K_{3}\right\},
$$


and combining with (4.48), (4.49), (4.51), and (4.52), we get

$$
\int_{|x| \geq K^{*}} \rho\left(\frac{|x|^{2}}{K^{2}}\right)\left|g\left(t, \theta_{-t} \omega, g_{0}\left(\theta_{-t} \omega\right)\right)\right|^{2} d x \leq 4 \epsilon,
$$

which completes the proof.

\section{Random Attractor}

In this section, we will prove the existence of a $\Phi$-random attractor for the $\operatorname{RDS} \varphi$ associated with the stochastic Gray-Scott system (3.8) on $\mathbb{H}$. According to Lemma 4.1 that $\varphi$ has a closed random absorbing set in $\boldsymbol{\Phi}$, which along with the $\boldsymbol{\Phi}$-pullback asymptotic compactness will obtain the existence of a unique $\boldsymbol{\Phi}$-random attractor. Next, we will establish the $\boldsymbol{\Phi}$-pullback asymptotic compactness of $\varphi$ by using the uniform estimate on the tails of solutions.

Lemma 5.1. Assume that $f \in \mathbb{H} \cap \mathbb{U}$. The $R D S \varphi$ is $\boldsymbol{\Phi}$-pullback asymptotically compact in $\mathbb{H}$, that is, for $\mathbb{P}$-a.e. $\omega \in \Omega$, the sequence $\left\{\varphi\left(t_{n}, \theta_{-t_{n}} \omega, g_{0, n}\left(\theta_{-t_{n}} \omega\right)\right)\right\}$ has a convergent subsequence in $\mathbb{H}$ if $t_{n} \rightarrow \infty$, where $\{B(\omega)\} \in \mathbb{\Phi}$ and $g_{0, n}\left(\theta_{-t_{n}} \omega\right) \in B\left(\theta_{-t_{n}} \omega\right)$.

Proof. Combining with Lemmas 4.1 and 4.4, one can find that the proof is slightly a modification of the proof of Lemma 5.2 in [22] (or see Lemma 5.1 in [20]). Thus, we omitted it here.

We are now in the position to state the main result

Theorem 5.2. Assume that $f \in \mathbb{H} \cap \mathbb{U}$. The random dynamical system $\varphi$ has a unique $\boldsymbol{\Phi}$-random attractor in $\mathbb{H}$.

Proof. Notice that $\varphi$ has a closed random absorbing set in $\Phi$ by Lemma 4.1 and the is $\Phi$ pullback asymptotically compact in $\mathbb{H}$ by Lemma 5.1. Hence, the existence of a unique $\boldsymbol{\Phi}$ random attractor in $\mathbb{H}$ follows from Proposition 2.6 consequently.

Remark 5.3. In the original three-component reversible Gray-Scott system (see [8]), the first constant $F$ appearing in the second variable $v$-section does not depend on the space variable $x$. Here we have to affiliate the constant $F$ to $f_{2}(x)$, or else, it will give a obstacle to establish the uniform estimates of solutions when $t \rightarrow \infty$. For example, when estimating the term $2 \int_{\mathbb{R}} \alpha(t) v f_{2} d x$, we have $2 \int_{\mathbb{R}} \alpha(t) v f_{2} d x \leq F\|v\|^{2}+\left(f_{2}^{2} \alpha^{2}(t) / F\right) \mid$ Dom $\mid$, where |Dom | denotes the volume of the domain. Actually, we know that it is maybe of infinite dimension on unbounded domain, which is different from the bounded case discussed in [9].

\section{Acknowledgments}

The author would like to thank the anonymous referee for very helpful comments and suggestions which largely improve the quality of the original paper. This work was supported by NSF of China Grant 11071165 and Guangxi Provincial Department of Research Project Grant 201010LX166. 


\section{References}

[1] L. Arnold, Random Dynamical Systems, Springer, Berlin, Germany, 1998.

[2] P. Gray and S. K. Scott, "Autocatalytic reactions in the isothermal, continuous stirred tank reactor. Isolas and other forms of multistability," Chemical Engineering Science, vol. 38, no. 1, pp. 29-43, 1983.

[3] P. Gray and S. K. Scott, "Autocatalytic reactions in the isothermal, continuous stirred tank reactor. Oscillations and instabilities in the system $a+2 b \rightarrow 3 b ; b \rightarrow c$," Chemical Engineering Science, vol. 39, no. 6, pp. 1087-1097, 1984.

[4] G. Nicolis and I. Prigogine, Self-Organization in Nonequilibrium Systems, John Wiley \& Sons, New York, NY, USA, 1977.

[5] I. Prigogine and R. Lefever, "Symmetry breaking instabilities in dissipative systems," The Journal of Chemical Physics, vol. 48, no. 4, pp. 1695-1700, 1968.

[6] S. K. Scott and K. Showalter, "Simple and complex reaction-diffusion fronts, chemical waves and patterns," in Understanding Chemical Reactivity, R. Kapral and K. Showalter, Eds., vol. 10, pp. 485-516, Springer, 1995.

[7] H. Mahara, N. J. Suematsu, T. Yamaguchi, K. Ohgane, Y. Nishiura, and M. Shimomura, "Three-variable reversible Gray-Scott model," Journal of Chemical Physics, vol. 121, no. 18, pp. 89688972, 2004.

[8] Y. You, "Dynamics of three-component reversible Gray-Scott model," Discrete and Continuous Dynamical Systems B, vol. 14, no. 4, pp. 1671-1688, 2010.

[9] A. Gu, "Random attractors for stochastic three-component reversible Gray-Scott system with multiplicative white noise," Journal of Applied Mathematics, vol. 2012, Article ID 810198, 15 pages, 2012.

[10] Y. You, "Dynamics of two-compartment Gray-Scott equations," Nonlinear Analysis. Theory, Methods E Applications, vol. 74, no. 5, pp. 1969-1986, 2011.

[11] J. M. Ball, "Continuity properties and global attractors of generalized semiflows and the NavierStokes equations," Journal of Nonlinear Science, vol. 7, no. 5, pp. 475-502, 1997.

[12] J. M. Ball, "Global attractors for damped semilinear wave equations," Discrete and Continuous Dynamical Systems A, vol. 10, no. 1-2, pp. 31-52, 2004.

[13] T. Caraballo, G. Łukaszewicz, and J. Real, "Pullback attractors for asymptotically compact nonautonomous dynamical systems," Nonlinear Analysis. Theory, Methods E Applications A, vol. 64, no. 3, pp. 484-498, 2006.

[14] I. Moise, R. Rosa, and X. Wang, "Attractors for noncompact nonautonomous systems via energy equations," Discrete and Continuous Dynamical Systems A, vol. 10, no. 1-2, pp. 473-496, 2004.

[15] K. Lu and B. Wang, "Global attractors for the Klein-Gordon-Schrödinger equation in unbounded domains," Journal of Differential Equations, vol. 170, no. 2, pp. 281-316, 2001.

[16] B. Wang, "Attractors for reaction-diffusion equations in unbounded domains," Physica D, vol. 128, no. 1, pp. 41-52, 1999.

[17] B. Wang, "Pullback attractors for non-autonomous reaction-diffusion equations on $\mathbb{R}^{n}$," Frontiers of Mathematics in China, vol. 4, no. 3, pp. 563-583, 2009.

[18] B. Wang, "Pullback attractors for the non-autonomous FitzHugh-Nagumo system on unbounded domains," Nonlinear Analysis. Theory, Methods E Applications A, vol. 70, no. 11, pp. 3799-3815, 2009.

[19] M. Prizzi, "Averaging, Conley index continuation and recurrent dynamics in almost-periodic parabolic equations," Journal of Differential Equations, vol. 210, no. 2, pp. 429-451, 2005.

[20] P. W. Bates, K. Lu, and B. Wang, "Random attractors for stochastic reaction-diffusion equations on unbounded domains," Journal of Differential Equations, vol. 246, no. 2, pp. 845-869, 2009.

[21] B. Wang, "Random attractors for the stochastic Benjamin-Bona-Mahony equation on unbounded domains," Journal of Differential Equations, vol. 246, no. 6, pp. 2506-2537, 2009.

[22] Z. Wang and S. Zhou, "Random attractor for stochastic reaction-diffusion equation with multiplicative noise on unbounded domains," Journal of Mathematical Analysis and Applications, vol. 384, no. 1, pp. 160-172, 2011.

[23] I. D. Chueshov, Monotone Random Systems Theory and Applications, vol. 1779, Springer, Berlin, Germany, 2002.

[24] P. W. Bates, H. Lisei, and K. Lu, "Attractors for stochastic lattice dynamical systems," Stochastics and Dynamics, vol. 6, no. 1, pp. 1-21, 2006.

[25] X. Fan, "Attractors for a damped stochastic wave equation of sine-Gordon type with sublinear multiplicative noise," Stochastic Analysis and Applications, vol. 24, no. 4, pp. 767-793, 2006. 
[26] T. Caraballo and K. Lu, "Attractors for stochastic lattice dynamical systems with a multiplicative noise," Frontiers of Mathematics in China, vol. 3, no. 3, pp. 317-335, 2008.

[27] A. Gu, S. Zhou, and Z. Wang, "Uniform attractor of non-autonomous three-component reversible Gray-Scott system," In press.

[28] F. Morillas and J. Valero, "Attractors for reaction-diffusion equations in $\mathbb{R}^{n}$ with continuous nonlinearity," Asymptotic Analysis, vol. 44, no. 1-2, pp. 111-130, 2005.

[29] P. Imkeller and B. Schmalfuss, "The conjugacy of stochastic and random differential equations and the existence of global attractors," Journal of Dynamics and Differential Equations, vol. 13, no. 2, pp. 215-249, 2001. 


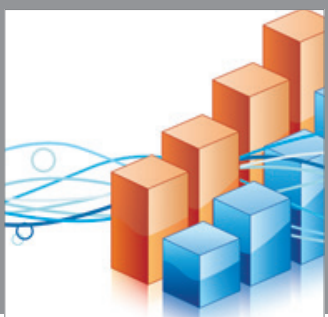

Advances in

Operations Research

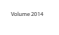

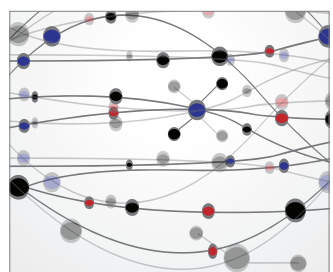

\section{The Scientific} World Journal
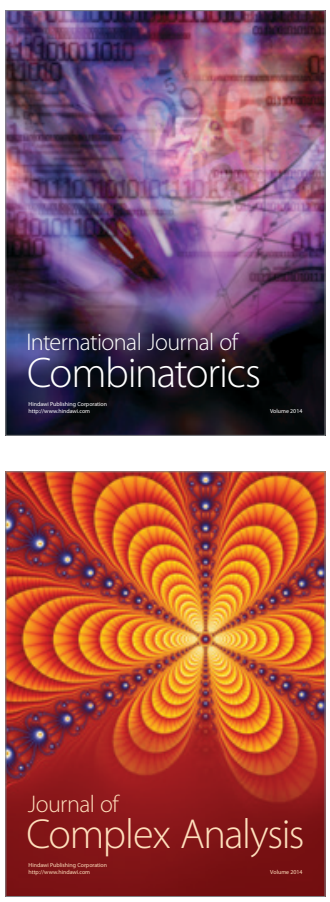

International Journal of

Mathematics and

Mathematical

Sciences
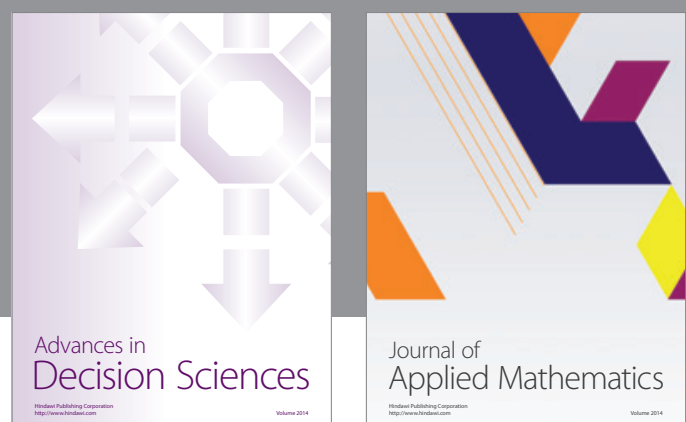

Journal of

Applied Mathematics
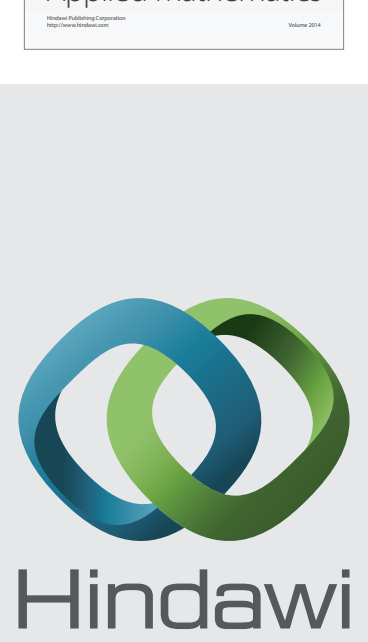

Submit your manuscripts at http://www.hindawi.com
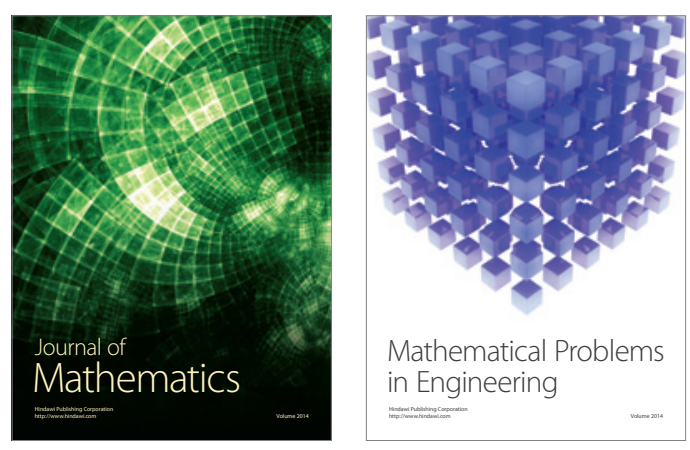

Mathematical Problems in Engineering
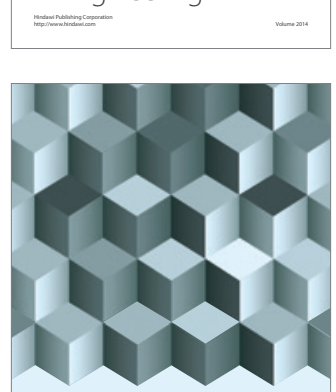

Journal of

Function Spaces
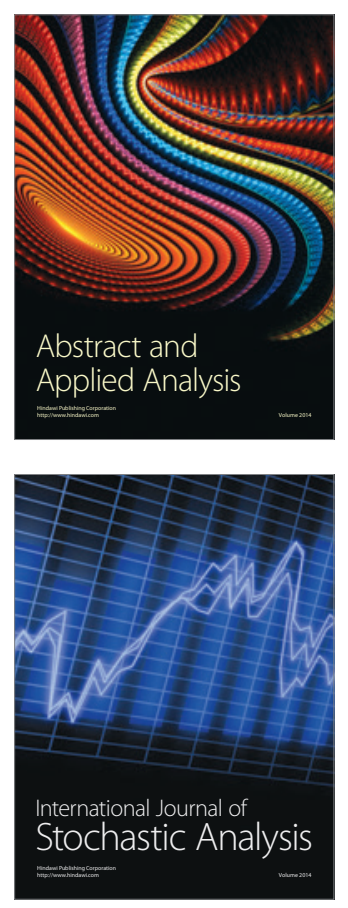

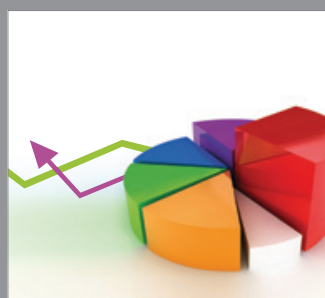

ournal of

Probability and Statistics

Promensencen
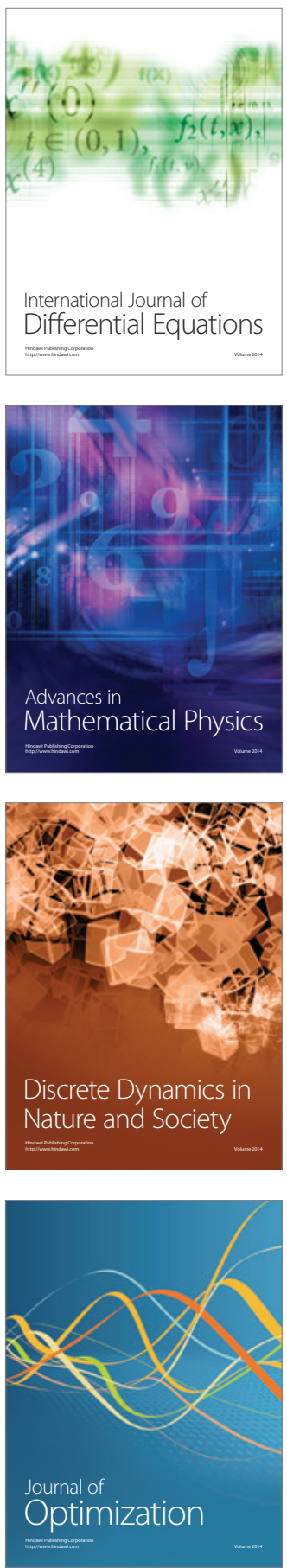